\title{
Distribution of Candida Species and Their Susceptibility to Antifungal Drugs in Dakar, Senegal
}

\author{
Khadime Sylla ${ }^{1,2, *}$, Roger Clement Tine ${ }^{1,2}$, Leon Amath Ndiaye ${ }^{1}$, Doudou Sow ${ }^{1,2}$, Souleye Lelo ${ }^{1,2}$, \\ Mamadou Dia ${ }^{2}$, Babacar Faye ${ }^{1}$, Therese Dieng ${ }^{1,2}$ \\ ${ }^{1}$ Department of Parasitology-Mycology, University Cheikh Anta Diop, Dakar, Senegal \\ ${ }^{2}$ Laboratory of Parasitology-Mycology, National University Hospital of Fann, Dakar, Senegal
}

\section{Email address:}

khadimesylla@yahoo.fr (K. Sylla), rogertine@hotmail.com (R. C. Tine), amath94@hotmail.com (L. A. Ndiaye),

doudsow@yahoo.fr (D. Sow), lelosouleye82@hotmail.com (S. Lelo), oudamamdia@gmail.com (M. Dia), bfaye67@yahoo.fr (B. Faye),

therese.dieng@ucad.edu.sn (T. Dieng)

${ }^{*}$ Corresponding author

\section{To cite this article:}

Khadime Sylla, Roger Clement Tine, Leon Amath Ndiaye, Doudou Sow, Souleye Lelo, Mamadou Dia, Babacar Faye, Therese Dieng. Distribution of Candida Species and Their Susceptibility to Antifungal Drugs in Dakar, Senegal. International Journal of Infectious Diseases and Therapy. Vol. 4, No. 4, 2019, pp. 50-55. doi: 10.11648/j.ijidt.20190404.11

Received: October 4, 2019; Accepted: October 24, 2019; Published: October 30, 2019

\begin{abstract}
The large spectrum of Candida species and their susceptibility to antifungal drugs has made the identification of Candida species and the detection of drug resistance necessary for the management of Candida infection. This study was carried out to determine the distribution of Candida species and to evaluate their susceptibility to antifungal drugs. A prospective observational and descriptive study was conducted from March to June 2016 in the laboratory of ParasitologyMycology at Fann University Hospital in Dakar. Samples were analyzed by direct microscopy and culture. Identification of Candida species was based on filamentation test, chlamydosporulation formation, auxanogramme (AUXACOLOR ${ }^{\mathrm{TM}} \mathrm{Bio}-\mathrm{Rad}$ ) and Candi-Select ${ }^{\circledR} 4$ (Bio-Rad,). The susceptibility of Candida species to antifungal drugs was tested using Fungitest ${ }^{\circledR}$ (BioRad) against 5-fluorocytosine, amphotericin B, miconazole, ketoconazole, itraconazole and fluconazole. A descriptive analysis was performed using Stata MP 14. Among 336 specimens received for mycological examination, 68 (20.2\%) were positive for Candida. The most identified Candida species were C. albicans (58.8\%), C. glabrata (16.2\%), C tropicalis (7.4\%), C krusei (7.4\%), C. parapsilosis (4.4\%), C. dubliniensis (4.4\%) and C. kefyr (1.5\%). The majority of isolates were susceptible to ketoconazole (94.3\%), fluconazole (85.7\%), amphotericin B and 5 fluoro-cytosine (88.6\%). The susceptibility rates were lower for itraconazole (51.4\%) and miconazole (68.6\%). One strain of $C$. albicans was resistant to 5 fluoro-cytosine, one strain of $C$. glabrata and C. tropicalis were resistant to itraconazole. The results of this study provide useful information regarding the distribution of Candida species and the susceptibility to antifungal drug. Routine identification of Candida species and monitoring of resistance patterns are necessary to manage Candida infection and to control the spread of resistance in clinical isolates of Candida species.
\end{abstract}

Keywords: Candida, Identification, Antifungal Susceptibility, Fungitest, Senegal

\section{Introduction}

Fungal species within genus Candida are known to colonize skin, nails, gastro-intestinal and vaginal mucosa. 20 to $25 \%$ of vaginal tract infections in women are due to Candida species [1-2]. Regarding the onychomycosis, $C$. albicans is the most frequent species causing Candida onychomycosis [3]. Overall, fungal infections are constantly increasing particularly fungal invasive infections. Over the last 20 years, the incidence of fungal invasive infection is highly increased and Candida species are identified as the main causal agent. In person with immune system deficiency, invasive candidiasis is the most frequent fungal infection. The most commonly isolated specie is Candida albicans $56 \%$. The mortality due to Candida albicans is about $37.9 \%$. Among the non-albicans species, 
C. glabrata, C. parapsilosis, C. tropicalis, C. krusei and C. dubliniensis have been identified as main etiologic agent [48].

The increased incidence and mortality related to invasive Candida infections (candidaemia) can be influenced by several factors such as characteristics of the population at risk (population age, HIV-positive, diabetes mellitus, nail traumatism, pregnancy, immunosuppressive and antibiotic therapy), standard of the healthcare facilities available, distribution of Candida species and prevalence of resistance $[7,9]$.

Correct identification of Candida species is necessary to confirm the etiological diagnosis and to guide the antifungal treatment. Providing adequate antifungal treatment is an essential component in the management of invasive Candida infection. Generally, the susceptibility of Candida albicans to azoles is well known. The primary resistance of Candida krusei to fluconazole and the possible resistance of Candida glabrata to fluconazole by the efflux mechanism are also demonstrated [10]. To avoid the emergence of resistance, it's important to identify the non-albicans Candida species in order to prescribe adequate treatment.

In Senegal, fungal infections due to Candida (onychomycosis, vaginal infection, invasive infection) are an important public health problem [10]. Data related to antifungal resistance are rare while fungal treatment is always prescribed in health facilities. Fluconazole, Itraconazole, Ketoconazole and Amphotericin B are the main fungal treatment prescribed to patients. Ketoconazole is currently removed from the list authorized drugs because of hepatotoxicity. In order to analyze the changing trends in the distribution of Candida species and to better guide clinician for the antifungal treatment prescription, we carried out this study aimed to determine the distribution of different Candida species and their susceptibility to six antifungal drugs (fluconazole, itraconazole, ketoconazole, miconazole, amphotericin B and 5-fluorocytosine).

\section{Materials and Methods}

\subsection{Study Design and Population}

A prospective observational and descriptive study was conducted from March to June 2016 in the laboratory of Parasitology-Mycology at Fann University Hospital in Dakar which is a mycological diagnostic reference center. All patients attending to the laboratory for a mycological examination, were included in this study.

\subsection{Laboratory Methods}

\section{Isolation and identification of Candida species}

All specimens were analyzed by direct microscopic examination. For the culture, specimens were inoculated both on Sabouraud-Chloramphenicol and on SabouraudChloramphenicol-Actidione medium. Incubation of these media was done at $37^{\circ}$ for 24 to 48 hours. Identification of
Candida species was based on macroscopic and microscopic examinations of cultures, filamentation test on serum, chlamydospore formation, AUXACOLOR ${ }^{\circledR}$ rapid identification system (Bio-Rad, France) and Candi-Select ${ }^{\mathbb{B}} 4$ (Bio-Rad, France).

\section{Antifungal susceptibility testing}

The study of the antifungal susceptibility of Candida isolates was performed using Fungitest ${ }^{\mathbb{B}}$ (Bio-Rad, France). Six antifungal agents tested were: 5-fluorocytosine, amphotericin $\mathrm{B}$, miconazole, ketoconazole, itraconazole and fluconazole.

Principle: Fungitest ${ }^{\circledR}$ is used to study the growth of yeasts in the presence of 6 antifungal agents at 2 different concentrations, in modified Roswell Park Memorial Institute, (RPMI) 1640 buffered medium, in the presence of a redox indicator. Growth assessment is based on reduction of the colored indicator which turns the medium from blue to pink. When growth is inhibited by the antifungal agent, the medium remains blue. This test, presented in the form of a 16 well microplate, consists of: (2 growth control wells), 12 wells containing the dehydrated antifungal agents (6 antifungal agents at 2 different concentrations) and (2 negative control wells). The breakpoints have been chosen following the study of the distribution of the antifungal agent's minimal inhibitory concentration (MIC) obtained with prototype microplates used with the same procedure as Fungitest $^{\circledR}[11]$.

Reading and Interpretation of results: (i) Only examine the plate when the positive control $(\mathrm{T}+)$ wells are pink. (ii) Observe any color change in the wells containing the antifungal agent compared to the negative control wells (blue). (iii) Interpret according to the color of the 2 wells for each antifungal agent: Blue-Blue $=$ no growth: strain inhibited by the antifungal agent in vitro, Pink-Blue=low growth: intermediate strain, Pink-Pink=growth: strain not inhibited by the antifungal agent in vitro [11].

\subsection{Statistical Methods}

After data collection, data were entered in Excel software and the analysis was performed using Stata software version MP 14. Quantitative variables were described in terms of means, standard deviation. For qualitative data, percentage was used to assess the frequency of each outcome with a $95 \%$ confidence interval (CI). Significance level of the different tests was 0.05 two-sided.

\subsection{Ethical Considerations}

This study was conducted in accordance with the Declaration of Helsinki. To respect the confidentiality, an identification code was assigned to each patient. This study was a hospital-based research conducted in routine conditions. The protocol was approved by the by the Research Ethic Committee (Comité d'Ethique et de Recherche: CER) of University Cheikh Anta Diop of Dakar (UCAD) (approval number: 48/2019/CER/UCAD). 


\section{Results}

\subsection{Mycological Data}

During the study period, 336 patients were enrolled in this study. The mean age was $34.08 \pm 12.4$ years. Study population was mainly constituted by patients aged between 25 to 35 years $(44.1 \%)$ and women $(95.6 \%)$ (Table 1$)$.

Table 1. General characteristics of study participants.

\begin{tabular}{|c|c|c|c|}
\hline & Frequency $(n=68)$ & Percentage (\%) & $95 \%$ CI \\
\hline \multicolumn{4}{|c|}{ Age group (years) } \\
\hline $14-25$ years & 18 & 26.5 & $15.6-41.8$ \\
\hline $25-35$ years & 30 & 44.1 & $29.7-62.9$ \\
\hline$>35$ years & 20 & 29.4 & $17.9-45.4$ \\
\hline \multicolumn{4}{|l|}{ Gender } \\
\hline Male & 3 & 4.4 & $0.9-12.8$ \\
\hline Female & 65 & 95.6 & $73.7-99.9$ \\
\hline \multicolumn{4}{|c|}{ Origin of specimens } \\
\hline Vaginal swab & 54 & 79.4 & $59.6-99.9$ \\
\hline Nails & 8 & 11.8 & $5.1-23.2$ \\
\hline Squama & 5 & 7.4 & $2.4-17.2$ \\
\hline $\begin{array}{l}\text { Auricular } \\
\text { specimen }\end{array}$ & 1 & 1.5 & $00-8.2$ \\
\hline
\end{tabular}

Among total specimens received for mycological examination, $68(20.2 \%)$ were found to be positive for Candida. The majority of Candida isolates came from a vaginal swab (79.4\%), nails specimens $(11.8 \%)$ and squama specimen $(7.4 \%)$.

The distribution of Candida species was as follows: $C$. albicans $58.8 \% \quad(\mathrm{n}=40) ; \quad$. glabrata $16.2 \% \quad(\mathrm{n}=11) ; C$ tropicalis $7.4 \%(\mathrm{n}=5)$; C krusei 7.4\% $(\mathrm{n}=5)$; C. parapsilosis $4.4 \%(\mathrm{n}=3) ;$ C. dubliniensis $4.4 \%(\mathrm{n}=3)$ and C. kefyr $1.5 \%$ $(\mathrm{n}=1)$ (Figure 1).

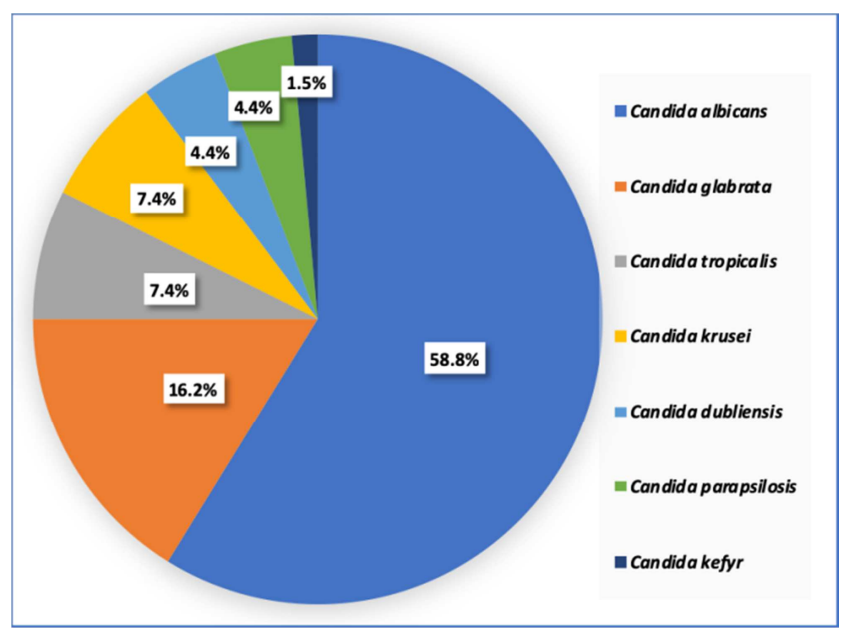

Figure 1. Distribution of Candida species.

\subsection{Antifungal Susceptibility}

Among 68 Candida strains isolated, 35 were tested for antifungal drugs: $C$. albicans $(n=20), C$. glabrata $(\mathrm{n}=6), C$. tropicalis $(\mathrm{n}=5), C$. parapsilosis $(\mathrm{n}=2)$ and C. krusei $(\mathrm{n}=2)$.

The majority of isolates were susceptible to ketoconazole (94.3\%), fluconazole (85.7\%), amphotericin B (88.6\%) and 5 fluoro-cytosine $(88.6 \%)$. The susceptibility rate was lower for itraconazole (51.4\%) and miconazole (68.6\%). Intermediate susceptibility was also described, and it was higher for itraconazole $(42.9 \%)$ and miconazole $(28.6 \%)$. $2.9 \%$ of resistance was observed for fluconazole, ketoconazole, miconazole and 5 fluoro-cytosine. It was 5.7\% for itraconazole and amphotericin B (Table 2).

Table 2. Percentage of susceptibility of antifungal agents by Fungitest (Bio-Rad) (\%).

\begin{tabular}{lllllll}
\hline & Fluconazole & Itraconazole & Ketoconazole & Miconazole & Amphotericin B & 5 Fluoro-cytosine \\
\hline Sensible & 85.7 & 51.4 & 94.3 & 68.6 & 88.6 \\
Intermediate & 11.4 & 42.9 & 5.7 & 28.6 & 5.7 \\
Resistant & 2.9 & 5.7 & 2.9 & 2.9 & 5.6 \\
\hline
\end{tabular}

C. albicans was susceptible to all antifungal drugs: $100 \%$ for fluconazole, ketoconazole, and amphotericin B, $80 \%$ for itraconazole and $90 \%$ for miconazole. Only one strain (5\%) was resistant to 5 fluoro-cytosine. The susceptibility of Candida glabrata was $100 \%$ for 5 fluoro-cytosine, $83.3 \%$ for fluconazole, ketoconazole, miconazole, amphotericin B. One strain of C. glabrata $(16.6 \%)$ was resistant to itraconazole and 4 strains $(66.6 \%)$. The majority of C. tropicalis $(80 \%)$ was susceptible to fluconazole, ketoconazole, amphotericin B and 5 fluoro-cytosine. One strain of $C$. tropicalis was resistant to all the azole and amphotericin $\mathrm{B}$

C. parapsilosis was susceptible to ketoconazole and 5 fluoro-cytosine but all strains had intermediate susceptibility $(100 \%)$ to itraconazole and miconazole. For all C. krusei, an intermediate susceptibility to fluconazole, itraconazole, miconazole and 5 fluoro-cytosine was noted. One C. krusei strains was resistant to amphotericin B (Table 3).

Table 3. Antifungal susceptibility of Candida species using Fungitest (Bio-Rad) (n, \%).

\begin{tabular}{|c|c|c|c|c|c|c|}
\hline & & C. albicans $(\mathrm{n}=20)$ & C. glabrata $(\mathrm{n}=6)$ & C. tropicalis $(\mathrm{n}=5)$ & C. $\operatorname{krusei}(\mathrm{n}=2)$ & C. parapsilosis $(\mathrm{n}=2)$ \\
\hline \multirow{3}{*}{ Fluconazole } & $\mathrm{S}$ & $20(100 \%)$ & $5(83.3 \%)$ & $4(80 \%)$ & $1(50 \%)$ & 0 \\
\hline & $\mathrm{I}$ & 0 & $1(16.7 \%)$ & 0 & $1(50 \%)$ & $2(100 \%)$ \\
\hline & $\mathrm{R}$ & 0 & 0 & $1(20 \%)$ & 0 & 0 \\
\hline \multirow{3}{*}{ Itraconazole } & $\mathrm{S}$ & $16(80 \%)$ & $1(16.7 \%)$ & $1(20 \%)$ & 0 & 0 \\
\hline & $\mathrm{I}$ & $4(20 \%)$ & $4(66.6 \%)$ & $3(60 \%)$ & $2(100 \%)$ & $2(100 \%)$ \\
\hline & $\mathrm{R}$ & 0 & $1(16.7 \%)$ & $1(20 \%)$ & 0 & 0 \\
\hline Kétoconazole & $\mathrm{S}$ & $20(100 \%)$ & $5(83.3 \%)$ & $4(80 \%)$ & $1(50 \%)$ & $2(100 \%)$ \\
\hline
\end{tabular}




\begin{tabular}{|c|c|c|c|c|c|c|}
\hline & & C. albicans $(\mathrm{n}=20)$ & C. glabrata $(\mathrm{n}=6)$ & C. tropicalis $(\mathrm{n}=5)$ & C. krusei $(\mathrm{n}=2)$ & C. parapsilosis $(\mathrm{n}=2)$ \\
\hline \multirow{4}{*}{ Miconazole } & $\mathrm{R}$ & 0 & 0 & $1(20 \%)$ & 0 & 0 \\
\hline & $\mathrm{S}$ & $18(90 \%)$ & $5(83.3 \%)$ & $1(20 \%)$ & 0 & 0 \\
\hline & I & $2(10 \%)$ & $1(16.7 \%)$ & $3(60 \%)$ & $2(100 \%)$ & $2(100 \%)$ \\
\hline & $\mathrm{R}$ & 0 & 0 & $1(20 \%)$ & 0 & 0 \\
\hline \multirow{3}{*}{ Amphotericin B } & $\mathrm{S}$ & $20(100 \%)$ & $5(83.3 \%)$ & $4(80 \%)$ & $1(50 \%)$ & $1(50 \%)$ \\
\hline & I & 0 & $1(16.7 \%)$ & 0 & 0 & $1(50 \%)$ \\
\hline & $\mathrm{R}$ & 0 & 0 & $1(20 \%)$ & $1(50 \%)$ & 0 \\
\hline \multirow{3}{*}{5 Fluoro-cytosine } & $\mathrm{S}$ & $19(95 \%)$ & $6(100 \%)$ & $4(80 \%)$ & 0 & $2(100 \%)$ \\
\hline & I & 0 & 0 & $1(20 \%)$ & $2(100 \%)$ & 0 \\
\hline & $\mathrm{R}$ & $1(5 \%)$ & 0 & 0 & 0 & 0 \\
\hline
\end{tabular}

Sensible: S; Intermediate: I; Resistant: T.

\section{Discussion}

The prevalence of fungal infection is highly increasing worldwide, particularly invasive fungal infection. Candida albicans is described as the most frequent etiological agent of candidemia but other non-albicans species have been reported as emerging causal agents.

The management of Candida infection required correct identification of Candida species in order to establish definitive etiological diagnosis and to guide the prescription of antifungal drugs. The objective of this study was to determine the spectrum of different Candida species, and their susceptibility to antifungal drugs.

The prevalence of Candida infection was $20.2 \%$. The main source of Candida was vulvovaginal infection (70.5\%) and onychomycosis (12.6\%). The frequency of Candida in vaginal swab and nail specimen was described by other authors. Candida species were found to be the principal source of vaginal infection $(33.3 \%$ prevalence) in women attending to the laboratory of Mycology in Fann university hospital [12]. Seck et al when studying the epidemiological profile of onychomycosis found Candida species as the main etiological agent [13].

In our study, $C$. albicans was the main specie $(58.8 \%)$ followed by $C$. glabrata (16.2\%); $C$ tropicalis $(7.4 \%) ; C$ krusei $(7.4 \%) ; C$. parapsilosis $(4.4 \%)$ and $C$. dubliniensis (4.4\%). Similar results were previously described in the same department. Dieng et al when assessing the distribution of Candida species found C. albicans $(52.75 \%)$, C. tropicalis (4.4\%), C. glabrata (4.4\%), C. dubliniensis (1.1\%) [14]. Sow et al, when using MALDITOF Mass Spectrometry for Candida species identification found similar trends: $C$. albicans $(\mathrm{n}=128)$, C. glabrata $(\mathrm{n}=27)$, C. tropicalis $(\mathrm{n}=24)$, C. krusei $(\mathrm{n}=5)$, C. parapsilosis $(\mathrm{n}=1)$ [15].

A strain of $C$. kefyr was found in our result. This was previously described by other authors in the same laboratory [14-15].

C. albicans (72.6\%), C. glabrata (14.5\%) and C. tropicalis (9.7\%) were the main Candida species isolated in Abidjan in a study conducted by Djohan et al in 2012 [16]. Similar results were also described in Cameroon by Kamga et al in 2012 [17]. Another study conducted in Abidjan have found C. glabrata as main specie followed by $C$. albicans and $C$. tropicalis [18]. Candida dubliensis was also described in strains from Abidjan and Cameroon [17-18].
The distribution of Candida species found in our study is similar to what was found in Morocco by Uwingabiye et al in 2012 [19]. It was also similar to the distribution in Iran and Kuwait [20-21].

The evaluation of the susceptibility of Candida strains using Fungitest ${ }^{\circledR}$ show that the majority of Candida species were susceptible to ketoconazole, fluconazole, amphotericin $\mathrm{B}$ and 5 fluoro-cytosine (88.6\%).

C. albicans is susceptible to all antifungal drugs but one isolate (5\%) is resistant to 5 fluoro-cytosine. Khosravi et al when assessing the in vitro susceptibility of Candida species to antifungal drugs found that Candida albicans was susceptible to amphotericin B, itraconazole, fluconazole and ketoconazole but only one strain was resistant de flucytosine [21]. Resistance of C. albicans to flucytosine was previously described in Senegal by Dieng et al in 2001 who showed $11.1 \%$ of resistance [22].

Our results regarding the resistance of C. albicans to flucytosine are not in line with what found in Abidjan by Djohan et al and Alfouzan et al in Kuwait who didn't show resistance of $C$. albicans to flucytosine [16, 21].

The susceptibility of $C$. glabrata to fluconazole, ketoconazole, miconazole and amphotericin B was similar $(83.3 \%)$. It was $100 \%$ for 5 Fluoro-cytosine. One strain of $C$. glabrata was resistant to itraconazole $(16.7 \%)$. Our result regarding the susceptibility of $C$. glabrata to fluconazole, amphotericin and 5 fluoro-cytosine was similar with what was noted by Alfouzan et al in Kuwait [20]. The findings of this study regarding the susceptibility of C. glabrata to 5 Fluoro-cytosine are not in line with what was found by Khosravi et al who found total resistant of all C. glabrata (4 strains) to flucytosine [21]. The resistant of C. glabrata to itraconazole was previously described in Spain by Miranda et al [23]. The primary resistance of $C$. glabrata to fluconazole described previously [10, 24-25], was not found in our study.

C. tropicalis was susceptible to fluconazole, ketoconazole, amphotericin $\mathrm{B}$ and 5 fluoro-cytosine. These were demonstrated by Bonouman et al in Abidjan and Khosravi et al in Iran but $C$. tropicalis was resistant to flucytosine $(66.7 \%)$ in Iran $[18,21]$. The susceptibility of $C$. tropicalis to fluconazole, amphotericin B and flucytosine was also described by Ozer et al in Turkey [26]. In previous study conducted in Senegal by Dieng et al, $C$. tropicalis had intermediate susceptibility to miconazole and one strain was resistant to ketoconazole [22]. A strain of C. tropicalis was 
resistant to itraconazole and miconazole. This was not previously described but intermediate susceptibility to miconazole was observed for $C$. tropicalis [21, 27]. Similar results concerning the susceptibility of $C$. parapsilosis to ketoconazole, 5 fluoro-cytosine, fluconazole, itraconazole and miconazole results were demonstrated by other authors [28-30].

A strain of $C$. krusei and $C$. tropicalis was resistant to amphotericin B. This result was not in line with what found by other authors who described a susceptibility of $C$. krusei to amphotericin B [26-27]. The primary resistance of $C$. krusei to fluconazole was not observed in our study [28].

This study is preliminary study on antifungal susceptibility testing in Senegal. Results from this study give an idea regarding the prevalence of resistance and will allow to implement effective strategies for the prophylaxis and treatment of humans with Candida infections. Based on these results, a surveillance system could be implemented to monitor the emergence of resistance. One of the weaknesses of the study is non-use of molecular methods (PCR) which will allow to make the differentiation between $C$. albicans and the closely related species like $C$. dubliniensis.

\section{Conclusions}

Overall, the results of this study provide useful information regarding the distribution of Candida species and their susceptibility to antifungal drugs even if these findings may not allow to give sufficient conclusion regarding the susceptibility profile of Candida strains. However, surveillance is needed in order to identify any change in the species distribution and the emergence of resistance. To better describe the distribution of Candida species and their susceptibility to antifungal drugs, other studies using molecular methods for identification and conventional antifungal testing methods (CLSI or EUCAST) is required.

\section{Abbreviations}

RPMI: Roswell Park Memorial Institute, MIC: Minimal Inhibitory Concentration; CI: Confidence interval, UCAD; University Cheikh Anta of Diop, CER: Comité d'Ethique et de Recherche.

\section{Declarations}

Ethics Approval and Consent to Participate

The protocol was approved by the by the Research Ethic Committee of University Cheikh Anta Diop od Dakar (approval number: 48/2019/CER/UCAD).

\section{Consent to Publish}

The funder has no role to play in the manuscript writing, editing, and decision to publish.

Availability of Data and Materials

Data of this study are available from the corresponding author upon reasonable request.

\section{Competing Interests}

The authors declare that they have no competing interests.

\section{Funding}

This study was supported by Fann Teaching Hospital through the operating annual budget of the laboratory.

\section{Authors' Contributions}

KS conceived and designed the study. LAN and MD monitored the data collection. LAN collected data in the site. KS analyzed the data. KS wrote the first draft of the manuscript. All authors read and approved the final manuscript.

\section{Acknowledgements}

Through this study, we acknowledge the study participants, the staff of the department of ParasitologyMycology at Fann Teaching Hospital.

\section{References}

[1] Achkar JM, Fries BC. Candida infections of the genitourinary tract. Clin Microbiol Rev 2010; 23: 253-73.

[2] Freydière AM, Guinet R, Boiron P. Yeast identification in the clinical microbiology laboratory: phenotypical methods. Med Mycol 2001; 39: 9-33.

[3] Faergemann J. The role of yeasts in onychomycosis. Mycoses 1996; 39: 223-4.

[4] Eloy O, Blanc V, Mallié M, Decousser J. W, Pina P, Allouch P. Y. Identification and antifungal susceptibility testing of two strains of Candida in 95 French hospitals. J Med Mycol, 2005; 15 (3), 117-126.

[5] Hazen KC. New and emerging yeast pathogens. Clin Microbiol Rev 1995; 8: 462-478.

[6] Clark TA, Hajjeh RA. Recent trends in the epidemiology of invasive mycoses. Curr Opin Infect Dis 2002; 15: 569-574.

[7] Eggimann P, Garbino J, Pittet D. Epidemiology of Candida species infections in critically ill non-immunosuppressed patients. Lancet Infect Dis 2003; 3: 685-702.

[8] Perlroth J, CHOI B, SPELlBERG B. Nosocomial fungal infections: epidemiology, diagnosis, and treatment. Med Mycol 2007; 45; 321-346.

[9] Hobson RP. The global epidemiology of invasive Candida infections is the tide turning? J Hosp Infect 2003; 55, 159 168 .

[10] Richter SS, Galask RP, Messer SA, Hollis RJ, Diekema DJ, Pfaller MA. Antifungal susceptibilities of Candida species causing vulvovaginitis and epidemiology of recurrent cases. J Clin Microbiol 2005; 43: 2155-62.

[11] Fungitest ${ }^{\circledR}$. Determination of the sensitivity of yeasts to antifungal agents. Bio-Rad, France, 60780. 
[12] Sylla K, Sow D, Lakhe NA, Tine RC, Dia M, Lelo S et al. Candidoses vulvovaginales au laboratoire de ParasitologieMycologie du Centre Hospitalier Universitaire de Fann, Dakar (Sénégal). Rev Cames Santé, 2018; 5 (2).

[13] Seck MC, Ndiaye D, Diongue K, Ndiaye M, Badiane AS, Sow $\mathrm{D}$, et al. Profil mycologique des onychomycoses à Dakar (Sénégal). J Mycol Med 2014; 24: 124-8.

[14] Dieng Y, Sow D, Ndiaye M, Guichet E, Faye B, Tine R, et al. Identification de trois souches de Candida africana au Sénégal. J Mycol Med 2012; 22: 335-40.

[15] Sow D, Fall B, Ndiaye M, Ba BS, Sylla K, Tine R, Lo AC, Abiola A, Wade B, Dieng T, Dieng Y. Usefulness of MALDITOF mass spectrometry for routine identification of Candida species in a resource-poor setting. Mycopathologia 2015; 180: 173-9.

[16] Djohan V, Angora KE, Vanga-Bosson AH, Konaté A, Kassi FK, Yavo W, et al. In vitro susceptibility of vaginal Candida albicans to antifungal drugs in Abidjan (Ivory Coast). J Mycol Med 2012; 22: 129-33.

[17] Kamga HY, Agem FK, Tegankam D, Toukam M, Sando Z, Somo RM. Sensibilité aux antifongiques des Candida spp isolés dans les candidoses digestives chez les sujets séropositifs au VIH à Yaoundé-Cameroun. Health Sci AND Dis 2014; 15 (3).

[18] Bonouman-Ira V, Angora E, Djohan V, Vanga-Bosson H, Sylla-Thanon K, Beourou S, et al. Profil de résistance des Candida non albicans à Abidjan en 2011. Revue Bio-Africa 2011, 9: 27-31.

[19] Uwingabiye J, Iken M, Zohoun AG, Boumhil L, Lemkhente $\mathrm{Z}$, Naoui $\mathrm{H}$, et al. Comparison of two antifungal susceptibility testing of Candida sp. isolates using agar diffusion method: Neo-sensitabs ${ }^{\circledR}$ tablets and Bio-rad ${ }^{\circledR}$ disks. J Mycol Med 2016; 26: 61-5.

[20] Alfouzan W, Dhar R, Ashkanani H, Gupta M, Rachel C, Khan ZU. Species spectrum and antifungal susceptibility profile of vaginal isolates of Candida in Kuwait. J Mycol Med 2015; 25: 23-8.
[21] Khosravi AR, Shokri H, Mansouri P, Katiraee F, Ziglari T. Candida species isolated from nails and their in vitro susceptibility to antifungal drugs in the department of Dermatology (University of Tehran, Iran). J Mycol Med 2008; 18: $210-5$.

[22] Dieng Y. Sensibilité aux antifongiques des souches de candida responsables de candidose oropharyngée chez des sujets vivant avec le VIH. Dakar Med 2001; 2: 835.

[23] Miranda-Cadena K, Marcos-Arias C, Mateo E, Aguirre JM, Quindós G, Eraso E. Prevalence and antifungal susceptibility profiles of Candida glabrata, Candida parapsilosis and their close-related species in oral candidiasis. Arch Oral Biol 2018; 95: 100-7.

[24] Sellami H, Ayadi A. Etude de la sensibilité des différentes espèces de Candida. Rev. Tun. Infectiol 2008; 2: 1-79.

[25] Develoux M, Bretagne S. Candidoses et levuroses diverses. EMC-Maladies infectieuses 2005; 2: 119-39.

[26] Özer TT, Durmaz S, Yula E. Antifungal susceptibilities of Candida species isolated from urine culture. J Infect and Chemother 2016; 22: 629-32.

[27] ElFeky DS, Gohar NM, El-Seidi EA, Ezzat MM, AboElew SH. Species identification and antifungal susceptibility pattern of Candida isolates in cases of vulvovaginal candidiasis. Alexandria J Med 2016; 52: 269-77.

[28] Paugam A. Le point sur l'antifongigramme. La Lettre de l'infectiologue 2010, 25: 222-227.

[29] Zhang L, Zhou S, Pan A, Li J, Liu B. Surveillance of antifungal susceptibilities in clinical isolates of Candida species at 36 hospitals in China from 2009 to 2013. Int J Infect Dis 2015 ; 33 : $1-4$.

[30] Das PP, Saikia L, Nath R, Phukan SK. Species distribution and antifungal susceptibility pattern of oropharyngeal Candida isolates from human immunodeficiency virus infected individuals. Indian J Med Res 2016; 143: 495. 